\title{
Sea State Estimation Using Model-scale DP Measurements
}

\author{
H. Brodtkorb, Astrid; Nielsen, Ulrik D.; J. Sørensen, Asgeir
}

Published in:

Proceedings of OCEANS '15

Publication date:

2015

Document Version

Publisher's PDF, also known as Version of record

Link back to DTU Orbit

Citation (APA):

H. Brodtkorb, A., Nielsen, U. D., \& J. Sørensen, A. (2015). Sea State Estimation Using Model-scale DP Measurements. In Proceedings of OCEANS '15 IEEE.

\section{General rights}

Copyright and moral rights for the publications made accessible in the public portal are retained by the authors and/or other copyright owners and it is a condition of accessing publications that users recognise and abide by the legal requirements associated with these rights.

- Users may download and print one copy of any publication from the public portal for the purpose of private study or research.

- You may not further distribute the material or use it for any profit-making activity or commercial gain

- You may freely distribute the URL identifying the publication in the public portal

If you believe that this document breaches copyright please contact us providing details, and we will remove access to the work immediately and investigate your claim. 


\title{
Sea State Estimation Using Model-scale DP Measurements*
}

\author{
Astrid H. Brodtkorb ${ }^{1}$ and Ulrik D. Nielsen ${ }^{1,2}$ and Asgeir J. Sørensen ${ }^{1}$
}

\begin{abstract}
Complex marine operations are moving further from shore, into deeper waters, and harsher environments. The operating hours of a vessel are weather dependent, and good knowledge of the prevailing weather conditions may ensure cost-efficient and safe operations. This paper considers the estimation of the peak wave frequency of the on-site sea state based on the vessel's motion in waves. A sea state can be described by significant wave height, peak wave frequency, wave direction, and often wind speed and direction are added as well. The signal-based algorithm presented in this paper is based on Fourier transforms of the vessel response in heave, roll and pitch. The measurements are used directly to obtain an estimate of the peak frequency of the waves. Experimental results from model-scale offshore ship runs at the Marine Cybernetics Laboratory (MCLab) at NTNU demonstrate the performance of the proposed sea state estimation algorithm.
\end{abstract}

\section{INTRODUCTION}

Most marine operations are highly dependent on the Captain's experience, and his ability to make correct decisions in stressful situations. In addition it is often difficult for operators to judge the sea state only by visual observations especially from large vessels. A decision support system (DSS) containing detailed information about the on-site sea state contributes to the Captain's decision making process. Developing such decision support tools has seen an increasing interest over the years.

The sea state can for instance be estimated using a wave rider buoy, wave radar, or satellite images of the ocean topology. Traditionally the wave rider buoy has been a provider of such data, contributing greatly to the weather forecasts offshore. However, these are at fixed locations, and most likely not able to provide information of the on-site sea state where the particular operation is taking place. Installing a wave radar is fairly costly, and the system itself requires careful tuning on a daily basis. [1] proposes an algorithm for predicting the short-crested sea state based on wave radar measurements. The algorithm estimates the local sea state, and in special conditions it has been used to recreate actual wave trains. However it is a computationally demanding algorithm which depends fundamentally on the radar measurements to be regularly calibrated, see [2] and [3]. Moreover, the algorithm requires independent radar measurements and, thus two radar systems are needed if navigation and sea state estimation should

*This work was supported by the Research Council of Norway through the Centres of Excellence funding scheme, project number 223254 AMOS.

${ }^{1}$ Centre for Autonomous Marine Operations (AMOS), Department of Marine Technology, Norwegian University of Science and Technology (NTNU), Otto Nielsens vei 10, 7491 Trondheim, Norway

2 DTU Mechanical Engineering, Technical University of Denmark, Kgs. Lyngby, Denmark happen simultaneously. Satellite images are dependent on low cloud cover for quality of the data gathered. Inevitably the cloud cover becomes thicker at times when the sea state estimate may be of greater interest.

Today, the majority of marine vessels are equipped with various sensors to measure operational state and performance such as global wave-induced motions, fuel consumption, hull girder stresses and geographical position. One example is DP ships which typically are installed with motion reference units, gyros and position measurement systems. In this sense, the marine vessels are indirectly equipped with sea state measuring systems, since the sensor measurements can be used to infer about the on-site sea state. Thus, the thought of using ships as wave buoys has been explored quite extensively the last 10-15 years, e.g. [4], [5], [6] and [7]. One proposed method is called the wave buoy analogy, where the ship motions in 6 degrees of freedom (DOF), or other global ship responses such as hull girder stresses, are transformed into the frequency domain, and an estimate of the wave spectrum is obtained by means of parametric or Bayesian modeling. This method takes the vessel's motion response amplitude operators (RAOs), also called transfer functions, into account when estimating the wave spectrum and/or associated sea state parameters such as the peak wave frequency $\omega_{p}$, significant wave height $H_{s}$ and relative wave direction $\beta$. As noted, the estimation method relies on both measurements - the measured signals - and transfer functions to model the theoretical relation between the waves and vessel responses. In this respect, the method is partly signal-based and partly model-based. However, for referencing from here on the term model-based will be used. This term is also useful to distinct the procedure from a signal-based method which is based solely on measured signals.

A signal-based approach has been applied in a hybrid controller which switches between candidate controllers based on an estimate of the peak wave frequency, see [8], [9], and [10]. The method does not consider the vessel's motion RAOs, and hence cannot estimate $H_{s}$ or $\beta$, but it has been shown to track a changing sea state reasonably well. This paper looks further into the properties of this particular algorithm.

Other types of signal-based methods exist, as shown in [11], [12], [13] and, although state-of-the-art techniques allows for peak wave frequency estimation only, theoretical studies have been initiated towards estimation of significant wave height $H_{s}$ and wave direction $\beta$ as well, see [14]. The development of a (complete) signal-based method for sea state estimation, including $H_{s}$ and $\beta$ would be considered a very important step improving planning and execution of 
marine operations including DP and transit.

This paper presents one signal-based estimation algorithm, and it is applied to measurements from a model-scale vessel controlled by a DP system. The vessel is freely floating, using the thrusters as sole means of keeping a constant position. Model-scale experiments are done of different cases including various relative wave directions $\beta$ and loading conditions in several sea states.

The paper is organized as follows: Section II gives a brief introduction to DP, as this is a required tool for collecting data to be used in the estimation method. Section III describes the signal-based sea state estimation method including a small example. The experimental setup as well as validation of the experimental results is presented in Section IV, before the estimation results are presented and discussed in Section V. Section VI concludes the paper.

\section{Dynamic Positioning of Marine Vessels}

A DP control system should control the vessel position and heading to a fixed setpoint or pre-determined track (low speed) solely by using the thrusters of the vessel [15]. This control objective can be expressed as

$$
\lim _{t \rightarrow \infty} \boldsymbol{\eta}^{L F}(t)-\boldsymbol{\eta}^{*}(t) \rightarrow \mathbf{0}
$$

where $\boldsymbol{\eta}^{L F}(t)$ is the low frequency vessel position vector, and $\boldsymbol{\eta}^{*}(t)$ is the desired position vector. Normally for a ship in DP the position in surge and sway and the heading angle are controlled. This means that the heave, roll and pitch can be seen as uninfluenced by the control system, and hence these degrees of freedom (DOF) can be used in sea state estimation, as done later in the paper. However, as shown in [16], this may not be the case for semisubmersibles with small water-plane-area where the roll and pitch may be influenced by the DP system. Normally the position measurement at time $t$ can be expressed as

$$
\mathbf{y}(t)=\boldsymbol{\eta}^{L F}(t)+\boldsymbol{\eta}^{W F}(t)+\mathbf{v}(t),
$$

where $\boldsymbol{\eta}^{L F}(t)$ is the low frequency vessel position vector induced by slowly varying forces from the environment and thrusters, $\boldsymbol{\eta}^{W F}(t)$ is the first order wave frequency motion and $\mathbf{v}(t)$ is the sensor noise vector.

$\boldsymbol{\eta}^{L F}(t)$ is not available directly from (2), so therefore an observer of the type Kalman filter or nonlinear passive observer, is required to provide a state estimate by filtering out the wave frequency motions, sensor noise, and estimating bias from slowly varying environmental forces and unmodeled dynamics. The wave frequency motion is filtered out to reduce unnecessary wear and tear on the propulsion system. Sensor-based observers such as IMU integration filters, see [17], [18] and model-based observers such as the extended Kalman Filter, see [19], [20] or passive nonlinear observers, see [21] are often applied to DP.

The control algorithm takes in the estimated vessel position and velocity vectors $\hat{\boldsymbol{\eta}}^{L F}, \hat{\boldsymbol{\nu}}$ from the observer and compares it with the desired vessel position $\boldsymbol{\eta}^{*}(t)$ to calculate a control input $\mathbf{u}$, here exemplified by a nonlinear PID controller:

$$
\begin{aligned}
\mathbf{u} & =-\mathbf{R}^{T}(\psi) \mathbf{K}_{p}\left(\hat{\boldsymbol{\eta}}^{L F}-\boldsymbol{\eta}^{*}\right)-\mathbf{K}_{d} \hat{\boldsymbol{\nu}} \\
& -\mathbf{R}^{T}(\psi) \mathbf{K}_{i} \int_{0}^{t}\left(\hat{\boldsymbol{\eta}}^{L F}-\boldsymbol{\eta}^{*}\right) d t,
\end{aligned}
$$

where $\mathbf{K}_{p}, \mathbf{K}_{d}, \mathbf{K}_{i}$ are the nonnegative proportional, derivative and integral gain matrices, respectively. The different parts required for the DP system to fulfill the control objective (1) are described in detail in [22], [23], [24].

\section{Signal-Based Sea State Estimation ALGORITHM}

The signal-based method presented in this paper, is solely based on the measurements, i.e. the procedure does not require knowledge of the vessel motion RAOs, and in this way it is a purely signal-based method. This is in contrast to the wave buoy analogy which is model-based; because of the need for RAOs. The present approach is based on Fourier transforms, where $N$ samples of heave, roll and pitch motion in the time domain are transformed to the frequency domain, and an estimate of the peak wave frequency $\hat{\omega}_{p}$ is computed based on the resulting response spectra of the three motion components heave, roll and pitch.

\section{A. Assumptions on Time Domain Vessel Response}

In small to relatively high sea states, linear theory is often sufficient to describe irregular wave-induced motions on marine vessels [25]. The first order wave-induced response $\eta^{W F} \in \mathbb{R}^{6}$ in irregular waves can be written as a finite sum of sinusoidal components with different amplitudes $\mathbf{a}_{j} \in \mathbb{R}^{6}$, frequencies $\omega_{j}$, wave numbers $k_{j}$, relative direction $\beta_{j}$, and random phases $\epsilon_{j} \in[0,2 \pi]$ :

$\boldsymbol{\eta}^{W F}=\sum_{j=0}^{J-1} \mathbf{a}_{j} \sin \left(\omega_{j} t-k_{j} x \cos \left(\beta_{j}\right)-k_{j} y \sin \left(\beta_{j}\right)+\epsilon_{j}+\boldsymbol{\gamma}_{j}\right)$.

$\gamma_{j} \in \mathbb{R}^{6}$ is the vector of phase of the low frequency position and phase of the RAOs. Here $\beta=0^{\circ}$ is head sea, and $\beta=$ $180^{\circ}$ is following sea.

The low frequency vessel motion is usually modeled as a mass-damper-spring system subject to forces from current, wind, and mean and slowly varying forces due to wave loads. For a vessel on DP the thrusters will produce mean and slowly varying forces to cancel those from the environment. Slowly varying forces are modeled as sinusoidal components with frequency $\left(\omega_{j}-\omega_{i}\right)$, and therefore:

$$
\boldsymbol{\eta}=\boldsymbol{\eta}^{L F}+\boldsymbol{\eta}^{W F}=\sum_{m=0}^{M-1} \mathbf{A}_{m} \sin \left(\omega_{m} t+\boldsymbol{\Gamma}_{m}\right)
$$

where $\omega_{m}$ spans both the low frequency and wave frequency regime, $\mathbf{A}_{m} \in \mathbb{R}^{6}$ is the vector of low frequency and wave frequency amplitudes, and $\boldsymbol{\Gamma}_{m} \in \mathbb{R}^{6}$ is the total phase shift vector relative to the wave elevation.

The Fourier transform approach requires that the response due to any irregular wave train can be described by (4), and that the wave-induced vessel motions are small and in steady 
state. The steady state assumption may be relaxed, see for instance [13].

\section{B. Fast Fourier Transform of Vessel Response}

The DP measurements (2) are sampled every $T>0$ seconds, and $N \in \mathbb{Z}_{>1}$ consecutive measurements are stored in a shift register with state $\chi=\left[\chi_{1}, \ldots, \chi_{N}\right]^{T} \in \mathbb{R}^{6 N}$, where $\chi_{k} \in \mathbb{R}^{6}, k \in\{0, \ldots, N-1\}$ are the stored measurements. The state component $\chi_{1}$ contains the most recent sample, and $\chi_{N}$ contains the least recent sample.

$$
\begin{aligned}
& \chi_{1}^{+}=\mathbf{y} \\
& \chi_{2}^{+}=\chi_{1} \\
& \quad \vdots \\
& \chi_{N}^{+}=\chi_{N-1}
\end{aligned}
$$

The fast Fourier transform (FFT) is applied to $\chi$ in order to generate the response spectra. The time for each sample is defined as $t_{k}=T k$ so that $t_{k}=\{0, \ldots, N k\}$. We want to determine a complex polynomial $\mathbf{q}(\chi) \in \mathbb{R}^{6}$ with $i=\sqrt{-1}$

$$
\mathbf{q}(\boldsymbol{\chi})=\sum_{n=0}^{N-1} \mathbf{c}_{n} e^{i n t_{k}}
$$

which interpolates $\boldsymbol{\eta}$ in (4), i.e. we need to find the coefficients $\mathbf{c}_{0}, \ldots, \mathbf{c}_{N-1}$ so that $\boldsymbol{\eta}_{k}=\mathbf{q}\left(\chi_{k}\right)$. The coefficients are found by the formula:

$$
\mathbf{c}_{n}=\frac{1}{N} \sum_{k=0}^{N-1} \chi_{k} e^{-i n t_{k}}, n=\{0, \ldots, N-1\}
$$

The real part of the spectral amplitude $\mathbf{S}_{\eta}(f) \in \mathbb{R}^{6 N}$ of the measurements are then given by

$$
\mathbf{S}_{\eta}(f)=\operatorname{Re}\left\{\mathbf{F}_{N}^{6} \boldsymbol{\chi}\right\}
$$

where $\mathbf{F}_{N}^{6} \in \mathbb{R}^{6 N \times 6 N}$ is the stacked $N \times N$ Fourier matrix for all 6 DOFs, i.e. all elements of $\mathbf{F}_{N}$ are multiplied by the $6 \times 6$ identity matrix. $\mathbf{F}_{N}$ has the form

$$
\mathbf{F}_{N}=\left[\begin{array}{cccc}
1 & 1 & \cdots & 1 \\
e^{-i t_{0}} & e^{-i t_{1}} & \cdots & e^{-i t_{N-1}} \\
e^{-i 2 t_{0}} & e^{-i 2 t_{1}} & \cdots & e^{-i 2 t_{N-1}} \\
\vdots & \vdots & \vdots & \vdots \\
e^{-i(N-1) t_{0}} & e^{-i(N-1) t_{1}} & \ldots & e^{-i(N-1) t_{N-1}}
\end{array}\right] .
$$

The FFT solves (8) by splitting the problem into even and odd parts, which reduces the number of operations from $O\left(N^{2}\right)$ for normal discrete Fourier transform to $O(N) \log _{2} N$. For more on Fourier transforms, see for instance [26].

In this setting it is more convenient to have the spectrum and frequencies related to angular frequency $\omega=2 \pi f$

$$
\mathbf{S}_{\eta}(\omega)=\frac{T}{\pi} \mathbf{S}_{\eta}(f) .
$$

The function $\Upsilon: \mathbb{R}^{6 N} \rightarrow \mathbb{R}_{\geq 0}$ operates on $\mathbf{S}_{\eta}(\omega)$, returning the peak frequencies in each DOF $\omega_{p, i}, i=\{1, . .6\}$. In this paper the estimate of the peak wave frequency is the average of the heave, roll and pitch peak frequencies

$$
\hat{\omega}_{p}=\frac{\omega_{p, 3}+\omega_{p, 4}+\omega_{p, 5}}{3} .
$$

In the case where the response has multiple peaks, the frequency corresponding to the largest is utilized. In different sea states the vessel responds little in some DOFs and more in others. To make sure that the algorithm is robust, the estimate is taken as the average of the heave, roll and pitch motions.

Figure 1 shows a time series of heave and the corresponding response spectrum when (8) and (9) are applied to the series. The measured incident wave spectrum is also shown.
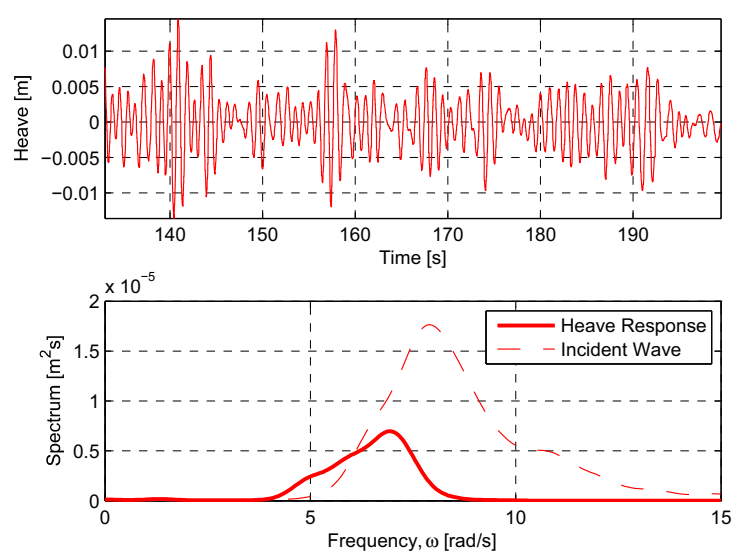

Fig. 1. Signal-based algorithm applied to a time series in heave; time series shown above and spectrum below. The spectrum of the heave response is plotted together with the FFT of the measured incident wave.

The response spectrum in each DOF is here found by using the Matlab toolbox WAFO [27], which is based on the builtin matlab function $\mathrm{f} f t$. See [10] to see how the estimation algorithm is implemented in a controller.

\section{EXPERIMENTAL SETUP AND VALIDATION OF MEASUREMENTS}

This section gives a brief introduction to the experimental facilities and presents the test cases. A thorough validation of the measurements was done, and it is also discussed.

\section{A. Vessel and Laboratory Facilities}

The Marine Cybernetics Laboratory (MCLab) at NTNU, Trondheim was used for the experiments. It includes a basin with dimensions $(L \times B \times D) 40 \mathrm{~m} \times 6.45 \mathrm{~m} \times 1.41 \mathrm{~m}$, a camera positioning system that provides position and orientation measurements to the DP system, and a wave flap ${ }^{1}$ for generating sea states from different wave spectra. Figure 2 shows the camera system and the model vessel in action.

The experiments were conducted with Cybership 3, a 1:30 scale model of a platform supply vessel (PSV) with dimensions $L_{p p}=1.971 \mathrm{~m}$ and $B=0.437 \mathrm{~m}$. It is equipped

${ }^{1}$ DHI Wave Synthesizer, www.dhigroup.com. 


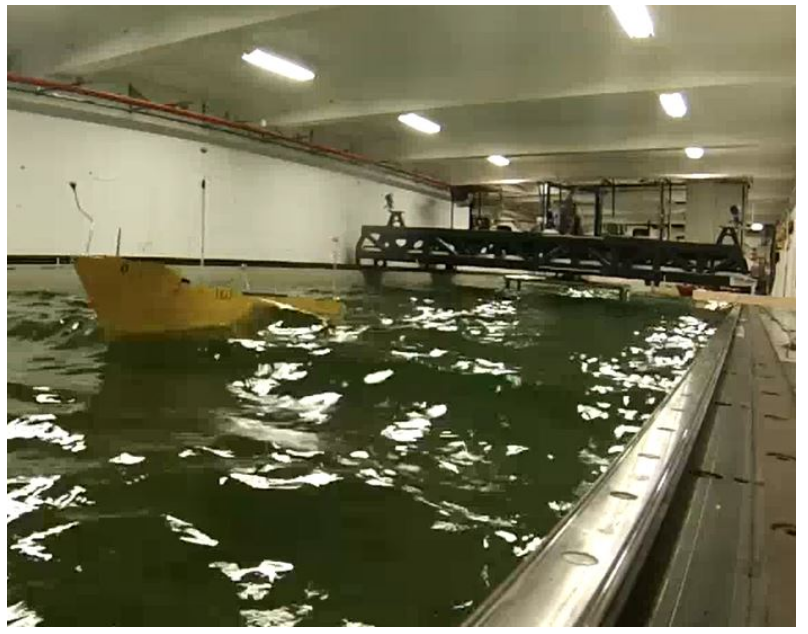

Fig. 2. Cybership 3 in action. The three cameras measuring position are seen above the bridge structure, and the surface elevation is measured to the far right at the wooden plank.

with three azimuth thrusters, two stern with fixed angles of $\pm 30^{\circ}$ and one in the bow at $90^{\circ}$, see Figure 3. The vessel has eight $12 \mathrm{~V}$ batteries supplying power to the thrusters and a National Instruments CompactRio (cRIO) where the DP control system is running. The operator supplies setpoints and specifies controller gains from a laptop, see Figure 4. Communication between the camera system, operator laptop and cRIO is via ethernet.

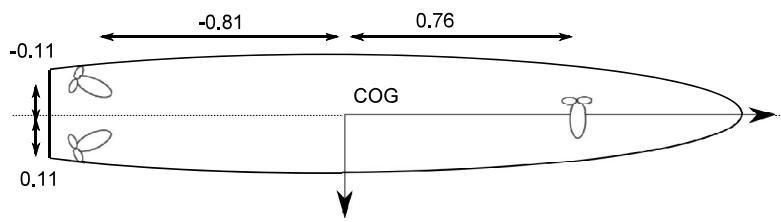

Fig. 3. Thruster configuration of Cybership 3.

\section{B. Experimental Test Cases}

Different cases were run with combinations of wave spectra with $\left(H_{s}, T_{p}\right)$, relative wave direction $\beta$ and loading conditions (LC), see Table I. The sea state numbers (SSn) are defined in Table II. The values within the parenthesis $\{\ldots\}$ in Table I are the ones changing in the case, e.g. for Case 1-3 the heading is changed for three different JONSWAP spectra J1, J2 and J3. Case a corresponds to $\beta=0^{\circ}$, Case b to $\beta=10^{\circ}$, and Case $\mathrm{c}$ to $\beta=20^{\circ}$.

Common wave spectra used in the North Sea include the wind-generated JONSWAP (Joint North Sea Wave Project)

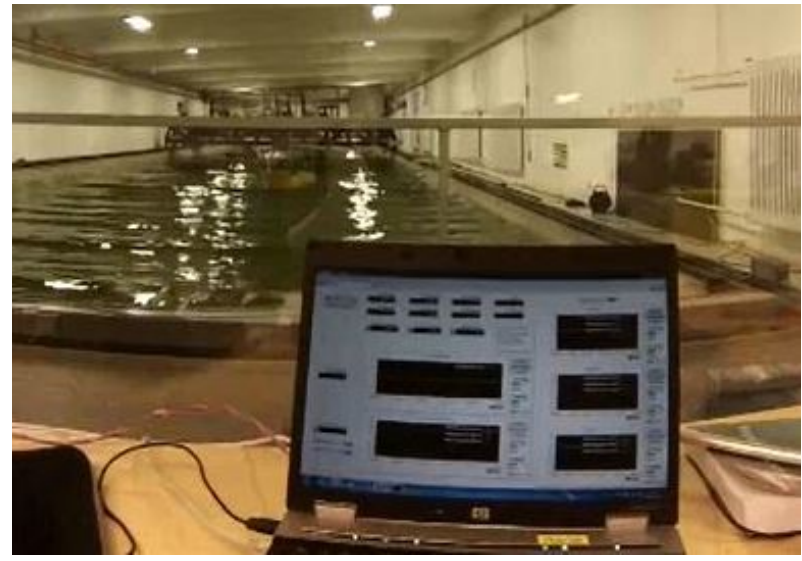

Fig. 4. Operator laptop where DP setpoints and controller gains are specified. The wave flap is also seen directly behind the laptop.

for developing sea states and the Pierson Moskovitz spectra for fully developed sea states, which are both single-peaked spectra. The Torsethaugen spectrum is also widely used to describe sea states in the North Sea. However, this is a double peaked spectrum and is not investigated in this paper. Experimental tests were done with a double-peaked spectrum: Case 4, but these results are omitted here, as further elaboration of the estimation method is needed to handle double-peaked spectra.

TABLE I

THE TEST CASES RUN IN MODEL-SCALE. LC = LOAD CONDITION; LC = 1 NORMAL, LC=2 8.6\% EXTRA WEIGHT.

\begin{tabular}{lccc} 
Case no. & SSn & $\beta\left[^{\circ}\right]$ & $\mathrm{LC}$ \\
\hline 1a,b,c & $\mathrm{J} 1$ & $\{0,10,20\}$ & 1 \\
2a,b,c & $\mathrm{J} 2$ & $\{0,10,20\}$ & 1 \\
3a,b,c & $\mathrm{J} 3$ & $\{0,10,20\}$ & 1 \\
$5 \mathrm{a}, \mathrm{b}, \mathrm{c}$ & $\mathrm{J} 1$ & $\{0,10,20\}$ & 2 \\
$5 \mathrm{~d}$ & $\mathrm{~J} 4$ & 0 & 2 \\
6a,b,c & $\{\mathrm{J} 1, \mathrm{~J} 2, \mathrm{~J} 3\}$ & 180 & 1 \\
7 & $\mathrm{~J} 4$ & 0 & 1 \\
8a,b,c & $\mathrm{PM} 1$ & $\{0,10,20\}$ & 1 \\
9a,b,c & $\{\mathrm{J} 1, \mathrm{~J} 2, \mathrm{~J} 3\}$ & 160 & 1 \\
10a,b & $\{\mathrm{J} 1, \mathrm{~J} 2\}$ & 30 & 1 \\
\hline \multicolumn{5}{r}{}
\end{tabular}

TABLE II

DEFINITION OF THE SEA STATE NUMBER (SSN) WITH SPECTER TYPE, $H_{s}$ AND $T_{p}$.

\begin{tabular}{clcc} 
SSn & Specter type & $H_{s}[\mathrm{~m}]$ & $T_{p}[\mathrm{~s}]$ \\
\hline J1 & JONSWAP & 0.04 & 0.8 \\
J2 & JONSWAP & 0.05 & 0.9 \\
J3 & JONSWAP & 0.05 & 1.5 \\
J4 & JONSWAP & 0.10 & 1.5 \\
PM1 & Pierson Moskovitz & 0.10 & 1.5 \\
\hline
\end{tabular}

\section{Validation of Measurements}

In this section the measurements of wave elevation and vessel response are validated. Two main issues relating to the measurements were found while processing the results, the first relates to the motion RAOs, and the second involves 
the sample time of the cRIO used for DP control and data logging.

1) Motion RAOs: Cybership 3 is a model made by MARINTEK in 1988, with RAO data from this time period as well. Since then the instrumentation onboard has changed, tha actual mass and mass distribution have changed as well. New RAOs were made by first finding the new center of gravity, the gyroradii and moments of inertia giving the mass distribution, waterline and trim. A ship lines plan was made from a rather coarse panel model, and the MARINTEK software Veres ShipX was used to calculate the motion RAOs in the center of gravity. The new motion RAOs have not been verified experimentally, although dedicated studies in this respect should be considered, as the effect of the coarse geometry file is unknown.

The response measurements of the vessel were taken at a point different from the center of gravity, so the new motion RAOs were translated to this point. The measurement point is $r=\left[x_{m}, y_{m}, z_{m}\right]=[110,0,153] \mathrm{mm}$ forward and above the center of gravity.

2) Sample Time of the cRIO: It was found that the cRIO did not manage to log with the specified sample time of $T=0.01 \mathrm{~s}$. The reason for the lag is that the model needed to wait for the other system loops in order to execute, and the total loop time of all control loops ${ }^{2}$ was $T=0.0133$ $\mathrm{s}$. As a result all logged measurements have sample time $T=0.0133 \mathrm{~s}$. For future tests it is advised to log the loop times of the individual control loops in addition to the system sample time.

Figure 5 shows a typical validation result for the measured surface elevation and pitch response. The top plot shows the wave spectrum specified in the wavemaker (dashed line) and the measured spectrum. The wave spectra have similar shapes and contain similar amounts of energy. The peaks are shifted slightly, and there are several possible reasons for this, for instance that the waves might not have been perfectly long-crested, the surface elevation was not measured at the center of the basin, reflections from the tank walls and wave beach, the tank filling, and calibration of the measurement device.

The middle plot shows the motion RAO for pitch for $\beta=0^{\circ}$. Pitch has two prominent resonance peaks at 4.9 and $7.7 \mathrm{rad} / \mathrm{s}$ with the lower having the higher amplitude. The resonance frequencies in heave are $6.2-6.9 \mathrm{rad} / \mathrm{s}$ for the different headings, and roll has one narrow peak at 6.7 $\mathrm{rad} / \mathrm{s}$. The shapes of the RAOs reveal that heave and pitch respond significantly to a broader range of frequencies than roll.

The bottom plot shows the pitch response spectrum obtained by the measured signal with corrected sample time (bold), and the theoretical response calculated based on the new motion RAO. It is observed that the frequency and magnitude of both peaks correspond reasonably well, though

\footnotetext{
${ }^{2}$ An illustration of the system architecture of the National Instruments Veristand Engine is found here: http://www.ni.com/productdocumentation/13033/en/
}
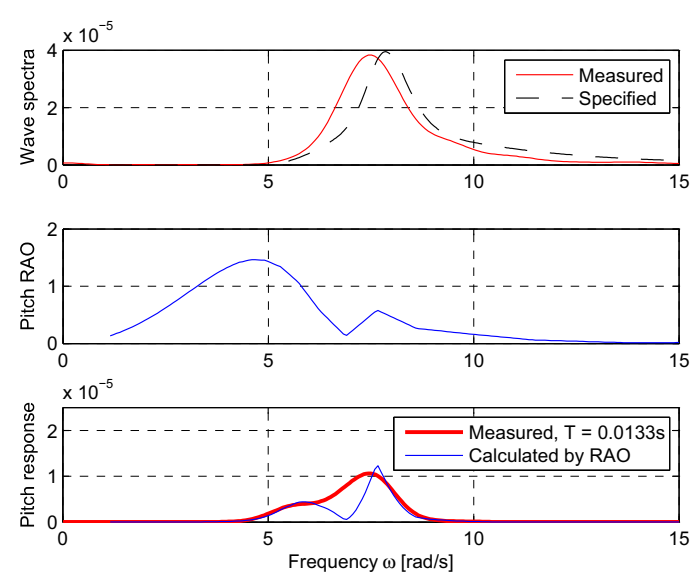

Fig. 5. Validation of the wave elevation from the JONSWAP J1 spectrum and pitch angle measurements for head sea $\beta=0^{\circ}$. Above: Measured and specified wave spectra, Middle: Pitch motion RAO for $\beta=0^{\circ}$, Below: Theoretical calculated response based on the RAO and the measured response with corrected sample time.

the cancellation effect at around $7 \mathrm{rad} / \mathrm{s}$ is not captured in the measurements.

Generally the frequencies of the peaks correspond very well, but the amplitudes are often off. Usually the amplitude of the measured response is higher than the theoretical calculated using RAOs, which means that the energy present at the different frequencies is larger in the experiment.

One explanation for this behaviour is that the DP system keeps the specified heading relative to the waves $\beta$ with an accuracy of around $\pm 3^{\circ}$. Since the motion RAO changes in amplitude with $\beta$, the theoretical and measured amplitudes do not match up. This is very prominent in roll, where the amplitude of the roll RAO more than doubles for each $10^{\circ}$ when $\beta \in\left\{0^{\circ}, 90^{\circ}\right\}$, with a similar decrease for $\beta \in\left\{90^{\circ}, 180^{\circ}\right\}$. The heave amplitudes generally agree more than illustrated by pitch in Figure 5, though the fit depends highly on the incident sea state. Alongside the DP system, a sea state that is not perfectly long-crested, may have the same effect on the response amplitudes.

In conclusion, the measurements correspond reasonably well with theory, and the observed deviations are justified.

\section{Estimation Results AND Discussion}

In this section the estimation results of the signal-based estimation algorithm applied to the measurements are presented and discussed.

\section{A. Results}

An example of typical response spectra is shown in Figure 6. The incident wave spectra (bold red) is from J3 (JONSWAP with $\left.\left(H_{s}, T_{p}\right)=(0.05,1.5)\right)$ with $\beta=20^{\circ}$, and the response in all DOFs are plotted, even though only heave, roll and pitch are used in the estimation. In the figure, the response peaks are grouped around two main frequencies, the wave, heave, pitch, surge and yaw around $4.2 \mathrm{rad} / \mathrm{s}$ and roll, heave and sway around $6.2 \mathrm{rad} / \mathrm{s}$. In the case of 
headings $\beta \neq 0^{\circ}$ there are large couplings. A summary of the estimation results is given in Table III presenting the arithmetic mean and standard deviation for each sea state. The error is the difference between the measured $\omega_{p}$ and each of $\omega_{p, 3}, \omega_{p, 4}, \omega_{p, 5}$ as well as $\hat{\omega}_{p}$, i.e for heave error $=\left(\omega_{p}-\omega_{p, 3}\right) / \omega_{p}$. Negative error means that the peak frequency from the response is higher than the incident peak wave frequency.

The following observations can be made:

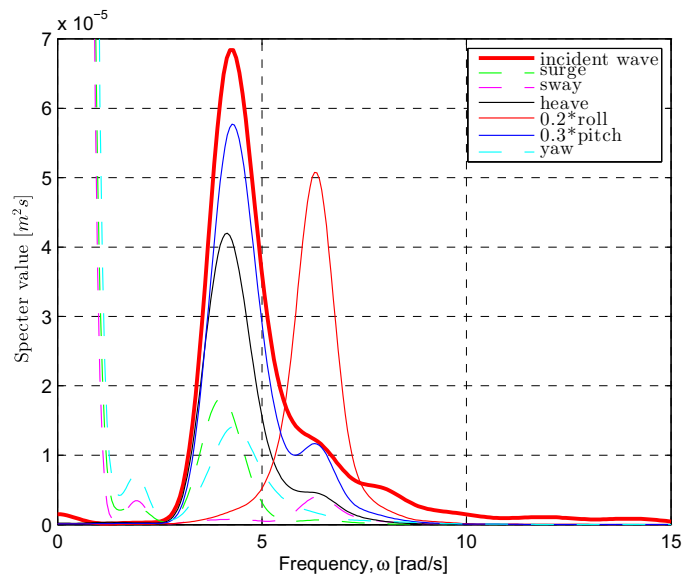

Fig. 6. Measured incident wave and measured response spectra for J3 (JONSWAP with $\left.\left(H_{s}, T_{p}\right)=(0.05,1.5)\right)$ with $\beta=20^{\circ}$.

TABLE III

SUMMARY OF THE RESULTS FOR $\hat{\omega}_{p}$ BASED ON $\omega_{p, 3}, \omega_{p, 4}, \omega_{p, 5}$, AND $\hat{\omega}_{p}=\operatorname{MEAN}\left(\omega_{p, 3}, \omega_{p, 4}, \omega_{p, 5}\right)$ FOR ALL DIRECTIONS AND LOADING CONDITIONS, SORTED BY SEA STATE (SEE TABLE II FOR DETAILS ON THE SEA STATES).

\begin{tabular}{lrccccc}
\multicolumn{6}{c}{ Measured and estimated peak wave frequency [rad/s] } \\
SSn & & $\omega_{p}$ & $\omega_{p, 3}$ & $\omega_{p, 4}$ & $\omega_{p, 5}$ & $\hat{\omega}_{p}$ \\
\hline J1 & mean & 7.545 & 6.938 & 6.444 & 7.086 & 6.823 \\
& std & 0.037 & 0.87 & 0.148 & 0.402 & 0.245 \\
& error & - & $8.0 \%$ & $14.6 \%$ & $6.1 \%$ & $9.6 \%$ \\
& & & & & & \\
J2 & mean & 7.056 & 6.655 & 6.332 & 6.615 & 6.534 \\
& std & 0.000 & 0.107 & 0.089 & 0.213 & 0.136 \\
& error & - & $5.7 \%$ & $10.3 \%$ & $6.3 \%$ & $7.4 \%$ \\
& & & & & & \\
J3 & mean & 4.244 & 4.101 & 6.209 & 4.255 & 4.855 \\
& std & 0.036 & 0.042 & 0.072 & 0.045 & 0.053 \\
& error & - & $3.4 \%$ & $-46.3 \%$ & $-0.3 \%$ & $-14.4 \%$ \\
& & & & & & \\
J4 & mean & 4.257 & 4.109 & 6.199 & 4.224 & 4.844 \\
& std & 0.038 & 0.063 & 0.087 & 0.025 & 0.058 \\
& error & - & $3.5 \%$ & $-45.6 \%$ & $0.8 \%$ & $-13.8 \%$ \\
& & & & & & \\
PM1 & mean & 7.517 & 6.411 & 6.334 & 6.151 & 6.299 \\
& std & 0.153 & 0.107 & 0.040 & 0.337 & 0.161 \\
& error & - & $14.7 \%$ & $15.7 \%$ & $18.1 \%$ & $16.2 \%$ \\
\hline
\end{tabular}

- Based on all 45 series: Pitch has the smallest absolute estimation error of $6.8 \%$, closely followed by heave with $7.4 \%$. Both these have fairly small standard deviations on the error, so they are the most reliable. Roll has the largest error and standard deviation of $22.2 \% \pm 14.8 \%$.
- Roll has the largest errors and standard deviations for all spectra except PM1, where pitch is worse. This behavior can be explained by inspecting the RAO. It is zero for small and high frequencies, and has one narrow peak at $6.7 \mathrm{rad} / \mathrm{s}$. Only wave components with frequency close to the natural frequency are transferred to the response, and hence wave spectra with peaks far away from the natural frequency in roll worsens the estimation, for instance when $T_{p}=1.5 \mathrm{~s}$.

- PM1 and J2 both have $\left(H_{s}, T_{p}\right)=(0.05,0.9)$, but $\hat{\omega}_{p}$ is very different. It is due to the wave spectrum shape; a spectrum with broader bandwidth will excite more frequencies in the response, and then the RAOs amplify a larger part of the incident wave spectrum. If the incident wave spectrum is narrow-banded, the response will naturally have smaller components outside the sea state bandwidth. Running experiments with spectra that have different peakedness would be very interesting.

- Roll estimates better for larger heading angles $\beta$, though not as well as heave and pitch. For the studied cases, better estimates would be obtained if roll was excluded. It could be interesting to see what happens for $\beta=90^{\circ}$.

- Pitch is marginally better than heave for head and following seas, and heave is marginally better than pitch for headings of $20,30,160^{\circ}$. This can again be related to the motion RAOs since the amplification in heave is larger when $\beta \in\left\{30^{\circ}-160^{\circ}\right\}$ and the opposite is observed in pitch.

- Case 1 and 5 have the same environmental conditions, but Case 5 has a $8.6 \%$ increase in mass. The estimation errors are slightly smaller for Case 1 than for Case 5. However, this may be a coincidence, as the difference in the measured peak frequency is large. The same timeseries of the wave elevation was run for these cases, so the difference may be due to measurement errors.

- Inspecting the spectra for surge, sway and yaw, see Figure 6 , the influence of the control system at low frequencies is noted, and the spectra do not go towards zero. This is because the thrusters insert energy at these frequencies. In the case where the observer does not filter out the wave frequency motion, the thrusters may also contribute to energy in the wave-frequency regime.

\section{B. Discussion with a Broader Perspective}

A topic that is always worth mentioning when modelscale tests are done is whether or not the findings have validity in full-scale. The vessel itself and the RAOs are scaled using Froude scaling, meaning that gravity forces like those exerted by non-breaking waves - are in theory scaled correctly. With Froude scaling, the vessel length and water depth are scaled by the ratio $\lambda=L_{f} / L_{m}$ ( $L_{f}$ is the full-scale length and $L_{m}$ is the model-scale length), and time is scaled by $\sqrt{\lambda}$. In particular in the MCLab, the water depth is only $1.5 \mathrm{~m}$, which scaled up to full-scale is 45 $\mathrm{m}$. The waves tested in the lab have full-scale heights of $H_{s, f}=1.2-3 \mathrm{~m}$ and peak periods of $T_{p, f}=4.38-8.21 \mathrm{~s}$, which puts the waves firmly within the shallow water regime. 
The thrusters and thrust losses should ideally be scaled by Reynold's scaling to get the viscous forces correct, but this is practically not possible to obtain simultaneously with Froude scaling. Effects from the DP system are probably larger than thrust scaling effects. So, all in all, the model-scale results are believed to be reasonably representative of expected behavior in full-scale.

For a vessel in transit or operation, there may be many frequencies of interest simultaneously: for instance the peak wave frequency, the encounter frequency in the case of forward speed, and the oscillation frequency of the different DOFs. These estimates are e.g. used in detection of parametric roll before it happens so that the vessel speed can be reduced, see [11], [12], active roll and pitch damping for instance in ship-platform gangways, heave compensation in cranes or risers, or directly in DP observers and controllers.

In most observers there is a wave filtering function. The filter contains a simple (synthetic) second order model of the wave motion of the vessel with a peak frequency and a damping ratio. The believed peak frequency of the waves is often applied here, see for instance [23], [22], [28], but perhaps inserting the vessel's oscillatory motions due to waves could yield even better filtering. The same sort of argument may be posed about hybrid controllers [8], [9], [10], where the controller is tuned according to the vessel's motions in a sea state, and not to the sea state directly.

\section{CONCLUSION}

The signal-based sea state estimation algorithm based on Fourier transforms presented in this paper was demonstrated to perform quite well in experiments with the model-scale offshore ship run at the Marine Cybernetics Laboratory (MCLab) at NTNU. It was found to be the most accurate using heave and pitch responses, and for narrow-peaked incident wave spectra.

Proposed further work includes more experiments with beam seas as well as with forward speed, and looking into sea states with different peakedness and double peaks.

\section{ACKNOWLEDGMENT}

A special thanks to Torgeir Wahl for helping out with the model experiments, and to Trond Innset and Lars Øien for helping with the calculation of new RAOs.

\section{REFERENCES}

[1] G. F. Clauss, S. Kosleck, and D. Testa, "Critical situations of vessel operations in short crested seas-forecast and decision support system," Journal of Offshore Mechanics and Arctic Engineering, vol. 134, no. 3, 2012.

[2] D. C. Stredulinsky and E. M. Thornhill, "Ship motion and wave radar data fusion for shipboard wave measurement," Journal of Ship Research, vol. 55, pp. 73-85, 2011.

[3] E. M. Thornhill and D. C. Stredulinsky, "Real Time Local Sea State Measurement using Wave Radar and Ship Motions," in SNAME annual meeting, Seattle, WA, USA, 2010.

[4] U. D. Nielsen, "Estimations of on-site directional wave spectra from measured ship responses," Marine Structures, vol. 19, pp. 33-69, 2006.

[5] T. Iseki and K. Ohtsu, "Bayesian estimation of directional wave spectra based on ship motions," Control Engineering Practice, vol. 8, pp. 215219, 2000.
[6] R. Pascoal and C. Guedes Soares, "Kalman filtering of vessel motions for ocean wave directional spectrum estimation," Ocean Engineering, vol. 36, pp. 477-488, 2009.

[7] E. A. Tannuri, J. V. Sparano, A. N. Simos, and J. J. Da Cruz, "Estimating directional wave spectrum based on stationary ship motion measurements," Applied Ocean Research, vol. 25, pp. 243-261, 2003.

[8] T. D. Nguyen, A. J. Sørensen, and S. T. Tong Quek, "Design of hybrid controller for dynamic positioning from calm to extreme sea conditions," Automatica, vol. 43, no. 5, pp. 768-785, 2007.

[9] T. D. Nguyen, A. J. Sørensen, and S. T. Quek, "Multi-operational controller structure for station keeping and transit operations of marine vessels," IEEE Transactions on Control Systems Technology, vol. 16, no. 3, pp. 491-498, 2008.

[10] A. H. Brodtkorb, A. J. Sørensen, and A. R. Teel, "Increasing the operation window of dynamic positioned vessels using the concept of hybrid control," ASME 2014 33rd International Conference on Ocean, Offshore and Arctic Engineering Volume 1A: Offshore Technology San Francisco, California, USA, June 8-13, 2014, 2014.

[11] D. J. W. Belleter, D. A. Breu, T. I. Fossen, and H. Nijmeijer, "A globally k-exponentially stable nonlinear observer for the wave encounter frequency," IFAC Proceedings Volumes (IFAC-PapersOnline), vol. 9, no. PART 1, pp. 209-214, 2013.

[12] D. J. W. Belleter, R. Galeazzi, and T. I. Fossen, "Experimental verification of a global exponential stable nonlinear wave encounter frequency estimator," Ocean Engineering, vol. 97, pp. 48-56, 2015.

[13] C. Møgster, Sea state estimation using Bayesian modeling methods, Department for Marine Teechnology, Norwegian University of Science and Technology (NTNU), 2015.

[14] U. D. Nielsen, M. Bjerregård, R. Galeazzi, and T. I. Fossen, "New concepts for shipboard sea state estimation," submitted to MTS/IEEE OCEANS'15 in Washington DC, 2015.

[15] D. N. V. (DNV), Rules for Classification of SHIPS Newbuildings, Special Equipment and Systems, Part 6 Chapter 7: Dynamic Positioning Systems. DNV (Det Norske Veritas) AS, Norway, 2010.

[16] A. J. Sørensen and J. P. Strand, "Positioning of small-waterplanearea marine constructions with roll and pitch damping," Control Engineering Practice, vol. 8, no. 2, pp. 205-213, 2000.

[17] B. Vik and T. I. Fossen, "Nonlinear observer design for integration of GPS and intertial navigation systems," Proceedings of the Conference on Decision and Control (CDC'2001). Orlando, FL., 2001.

[18] J. A. Farrell, T. D. Givargis, and M. J. Barth, "Real-time differential carrier phase GPS-aided INS," IEEE Transactions on Control Systems Technology, vol. 8, no. 4, pp. 709-721, 2000.

[19] E. A. Tannuri and H. M. Morishita, "Experimental and numerical evaluation of a typical dynamic positioning system," Applied Ocean Research, vol. 28, no. 2, pp. 133-146, 2006.

[20] V. Hassani, A. J. Sørensen, and A. M. Pascoal, "A novel methodology for robust dynamic positioning of marine vessels: Theory and experiments," Proceedings of the American Control Conference, pp. 560-565, 2013.

[21] T. I. Fossen and J. P. Strand, "Passive nonlinear observer design for ships using Lyapunov methods: full-scale experiments with a supply vessel," Automatica, vol. 35, no. 1, pp. 3 - 16, 1999.

[22] A. J. Sørensen, Marine Control Systems, Propulsion and Motion Control of Ships and Ocean structures, Lecture Notes. Department of Marine Technology, NTNU, 2013.

[23] T. I. Fossen, Handbook of Marine Craft Hydrodyamics and Motion Control. Wiley, 2011.

[24] A. J. Sørensen, J. P. Strand, and H. Nyberg, "Dynamic positioning of ships and floaters in extreme seas," Oceans Conference Record (IEEE), vol. 3, pp. 1850-1855, 2002.

[25] O. M. Faltinsen, Sea Loads on Ships and Offshore Structures. Cambridge University Press, 1993.

[26] E. Kreyszig, Advanced Engineering Mathematics, 9. ed. John Wiley \& sons, inc., 2006.

[27] P. A. Brodtkorb, P. Johannesson, G. Lindgren, I. Rychlik, J. Rydén, and E. Sjö, "WAFO - a Matlab toolbox for the analysis of random waves and loads," in Proc. 10'th Int. Offshore and Polar Eng. Conf., ISOPE, Seattle, USA, vol. 3, 2000, pp. 343-350.

[28] J. P. Strand and T. I. Fossen, "Nonlinear passive observer design for ships with adaptive wave filtering," vol. 244. Springer London, 1999, pp. 113-134. 